\title{
Conditions for Reticulated Crystalline Doping of Polymer with Charge Transfer Complexes
}

\author{
A. TRACZ, J. ULAŃSKI, and M. KRYSZEWSKI \\ Polymer Institute, Technical University of Łódź, \\ Żwirki 36, 90-924 Lódź, Poland
}

(Received February 28, 1983)

\begin{abstract}
Crystallization of the conductive charge transfer (CT) complex forming a reticulated, dendritic morphology within a polymeric matrix during film casting is a new way for obtaining conductive polymers. Subject to proper crystallization conditions during film casting one can obtain materials with high conductivity at a concentration of additives as low as $1 \mathrm{wt} \%$. This paper describes a model concept allowing for the selection of proper crystallization conditions leading to suitable morphology of crystalline additives. It is based on an analysis of morphologies from evaporating solutions containing polymer and low molecular weight CT complexes-forming additives. Considerations are based on the results obtained for TTF-TCNQ CT complexes crystallized in polycarbonate and polystyrene matrices.

KEY WORDS Charge Transfer Complexes / Organic Metalls / Conductivity of Polymers / Doping of Polymers / Crystallization in Polymeric Matrices /
\end{abstract}

In our previous paper it was shown that the crystallization of a low molecular weight conductive charge transfer (CT) complex in a polymer matrix during film casting leading to the formation of a reticulated crystalline morphology, is a new method for obtaining conductive polymeric systems. ${ }^{1}$ By choosing the proper conditions of film casting with simultaneous CT complex-crystallization, one can prepare a conductive material at a concentration of CT complex as low as $1 \mathrm{wt} \%$. In the case of polycarbonate (PC) doped with tetratiotetracene-tetracyanoquinodimethane (TTTTCNQ) a specific conductivity $10^{-2}(\Omega \mathrm{cm})^{-1}$ was obtained whereby the reticulated crystalline complex concentration did not exceed $1 \mathrm{wt} \%$. Thus the polymeric properties (e.g., mechanical properties) of the system were preserved. ${ }^{2}$

The proper conditions elaborated for the preparation of conducting polymer films consisting of PC and TTT-TCNQ were insufficient for systems with the best-known conducting CT complex: tetratiofulvalene (TTF)-TCNQ. This is probably due to the different solubility and crystallization ability of TTF-TCNQ compared with TTT-TCNQ complex. This prompted us to study the casting con- ditions under which the dopant (CT complex) crystallizes in nonequilibrium from (e.g., long thin wiskers or dendrites) to produce a conductive network penetrating the polymer matrix. This is a prerequisite for good film conductivity.

For minimization of the dopant amount, some local alignment and correlation between the neighbouring microcrystals is required since for a purely statistical dispersion, the amount of conducting filler must be high. ${ }^{3}$

The choice of appropriate conditions for conducting film preparation is complicated owing to many interdependent factors, affecting the crystallization process such as temperature, solubility of the CT complex and of polymer (solvent) quality, rate of solvent evaporation, viscosity of the solution (which increases continuously during solvent evaporation) and additive-polymer and additive-solvent interactions. All these factors were taken into consideration to formulate a general model concept for describing the conditions necessary to obtain a reticulated crystalline complex within an inert polymer matrix. This concept is based on comparative studies on the preparation and morphology of conducting films consisting of polycarbonate and 
polystyrene doped with TTT-TCNQ and TTFTCNQ complexes, the latter being particularly important.

\section{EXPERIMENTAL}

In studying the crystallization of TTF-TCNQ complex in polymeric matrices, the following polymers were used: poly(bisphenol A) carbonate (PC) and polystyrene (PS) and solvents: o-dichlorobenzene, chlorobenzene, dichloromethane and 1,2-dichloroethane. All low molecular weight compounds and solvents were used after standard purification (distillation or recrystallization). Polymers were purified by dissolution and precipitation from good solvents. The molecular weights of PC and PS were approximately 40,000 and 100,000, respectively. Both polymers were relatively inert and possible interactions between additives and polymeric matrices were not taken into consideration.

Hot solutions of $4 \mathrm{wt} \%$ polymer and $0.08 \mathrm{wt} \% \mathrm{CT}$ complex (with donor to acceptor molar ratio $1: 1$ ) were cast onto a glass substrate. For each solvent, several experiments were performed at different substrate temperatures (cast temperatures) ranging from room temperature to the boiling temperature of the given solvent. Thin films ( $c a .20 \mu \mathrm{m}$ ) obtained under different conditions were investigated by optical microscopy. The electrical conductivity of the obtained films was measured using the four probes method. The cast solution concentrations were measured as a function of solvent evaporation time at different temperatures. The viscosity of each so-

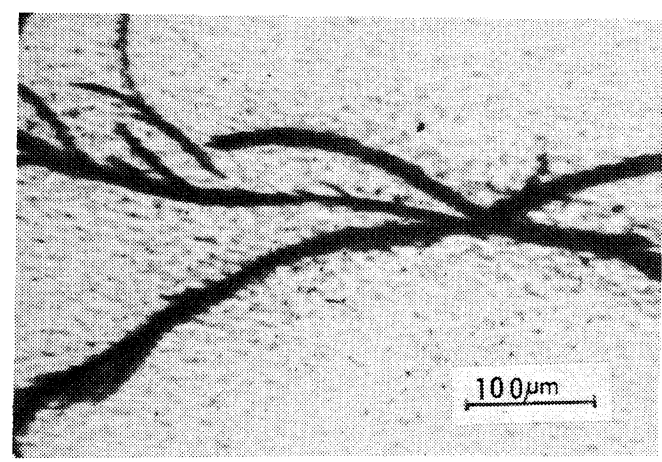

(a) lution was then determined at different temperatures using a Höppler viscometer. The obtained $\eta_{\mathrm{F}}=f(t)$ functions, corresponding to the progress of solution thickening were used to construct the schemes shown in Figures 2 and 3. These schemes depict only the general trends of viscosity increase and have qualitative meaning, the viscosities of the cast solutions could be determined only at the beginning of the casting procedure and not in the last stage just before the final film solidification.

\section{RESULTS AND DISCUSSION}

Typical micrographs of PC film samples containing the crystalline complex TTF-TCNQ prepared from chlorobenzene solutions at different casting temperatures are shown in Figure 1. It can be seen that the precipitated crystallites do not form a continuous network. At higher casting temperatures the complex TTF-TCNQ does not precipitate at all. The films obtained from other investigated solvents showed similar morphological structure. The same can be said for the morphology of crystallites formed in a PS matrix-separated aggregate of crystallites which are distributed within the film volume.

The microscopic observations of films prepared at different conditions make it possible to propose a model concept for the crystallization course of low molecular weight additives in polymer containing solutions. Let us consider first the time dependence of viscosity changes of cast solutions accompanied by simultaneous CT complex concentration increase. The function $\eta_{\mathrm{F}}=f(t)$ for different temperatures is schematically drawn in Figure 2. These

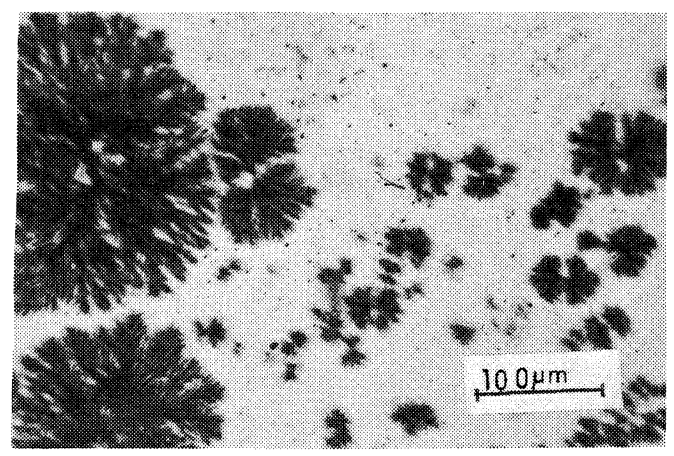

(b)

Figure 1. Micrograph of a PC film containing $2 \mathrm{wt} \%$ of TTF-TCNQ obtained from chlorobenzene; the casting temperature were $55^{\circ} \mathrm{C}$ (a) and $75^{\circ} \mathrm{C}$ (b). 


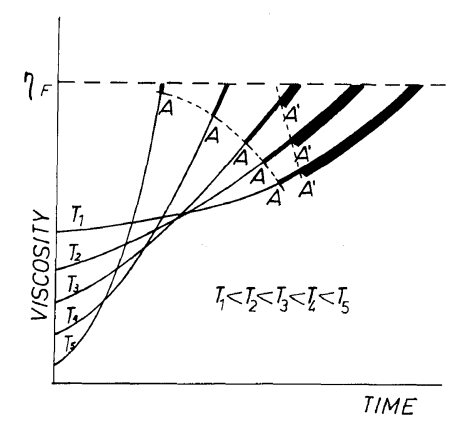

Figure 2. Scheme of solution viscosity $\eta_{F}$ dependence on evaporation time at different temperatures. The shape of the viscosity curves at different temperatures corresponds to that obtained experimentally.

curves may represent a situation in which chlorobenzene is used as a solvent. Let assume further that the point $\mathrm{A}$ represents the moment (due to solvent evaporation) at which the concentration of the TTT-TCNQ in solution reaches its critical value at the given temperature, causing crystallization to begin. For the system TTF-TCNQ, the corresponding points $\mathrm{A}^{\prime}$ are also indicated. Because of the better solubility and lower thermal stability of this complex, these points shift more than those for the TTT-TCNQ system. The viscosity values $\eta_{\mathrm{F}}$ correspond to a concentration at which the diffusion of low molecular weight additives of the crystallizable complexes is very slow, thus precluding the further growth of the crystallites.

At lower casting temperatures crystallization begins relatively early and because of the slow rate of solvent evaporation at these temperatures, the solution has a low viscosity for a long time period causing the crystallites to take on poorly branched, relatively large approximately the same as the equilibrium crystallites forms. In Figure 2 this situation is illustrated by curves $T_{3}$ and $T_{2}$ for complexes with TTT and TTF respectively. A typical micrograph obtained for films prepared under these conditions is shown in Figure 1a. The regions in which the crystalline complexes were found to be clustered and separated from each other when investigated by the local contact technique exhibited only high conductivity perpendicular to the surface. At lower temperatures, the conditions for forming proper morphological bases of additives are even more unfavourable. It may be deduced that the solvent evaporation rate should be higher for

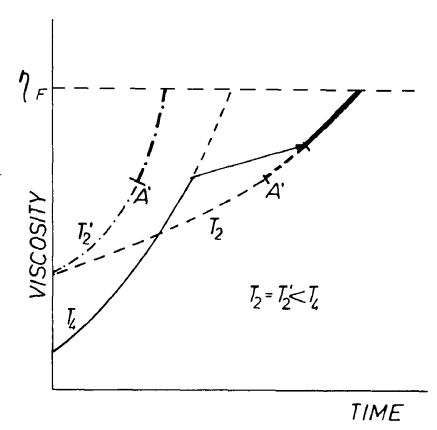

Figure 3. Scheme of solution viscosity dependence on temperature showing the effect of rapid cooling $\left(T_{4}, T_{2}\right)$ and pressure reduction $\left(\mathrm{T}_{2}{ }^{\prime}\right)$.

achieving considerable supersaturation of solution in a shorter time to result in rapid nonequilibrium crystallization.

The solvent evaporation rate can be accelerated by an increase in the casting temperature. However this temperature must not be too high because, due to the better solubility of additives at higher temperatures, crystallization would begin too late, as can be seen from curves $T_{5}$ and $T_{4}$ for complexes with TTT and TTF respectively. A rapid increase in the viscosity of the solution hinders the diffusion of components of the CT complex component and due to a short period of time before definitive film solidification, only a small portion of the additives can form crystalline precipitations. There may possibly be an intermediate cast temperature at which conditions are favourable for reticulated crystallization of the CT complex.

In the case of the PC + TTT-TCNQ system, when chlorobenzene was used as the solvent, the optimum cast temperature was about $100^{\circ} \mathrm{C}$ (this is relate to curve $\mathrm{T}_{4}$ in Figure 2). If $o$-dichlorobenzene is used as the solvent, the temperature is about $120^{\circ} \mathrm{C} .{ }^{1}$

In the case of PC+TTF-TCNQ a desirable morphological structure at any cast temperature could not be obtained. It is difficult to explain the difference in the crystallization conditions of TTTTCNQ and TTF-TCNQ in polymer matrices of the many factors effecting nucleation and further crystal growth. The phenomenological elucidation can be found from the scheme presented in Figure 2. At the temperatures at which precipitation of TTFTCNQ crystalls is possible (i.e., in the range from $T_{1}$ to $T_{2}$ on Figure 2) the solvent evaporation rate is too slow to achieve the required supersaturation in 


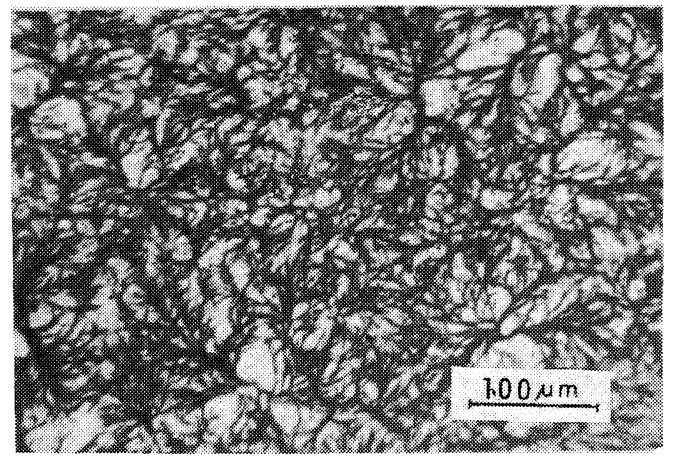

(a)

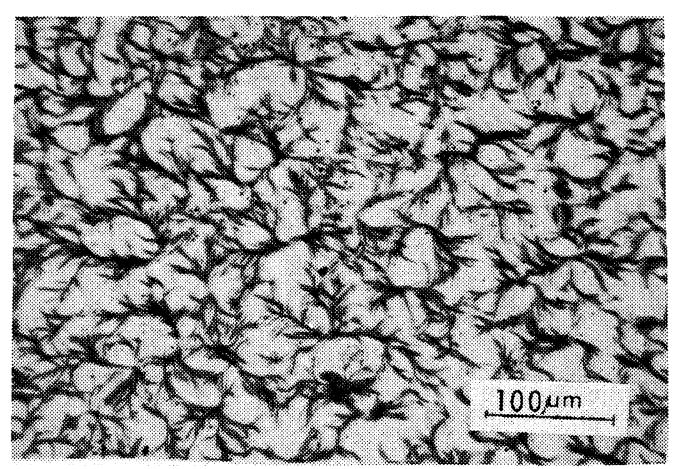

(b)

Figure 4. Micrograph of PC film (a) and PS film (b) containing $2 \mathrm{wt} \%$ of TTF-TCNQ obtained from chlorobenzene solution. Casting temperature $30^{\circ} \mathrm{C}$ after rapid cooling from $100^{\circ} \mathrm{C}$.

a short period of time. An appropriate rate of evaporation can be obtained at temperature $T_{4}$ (for chlorobenzene $c a .100^{\circ} \mathrm{C}$ ) but at this temperature TTF-TCNQ does not crystallize at all, probably due its higher solubility compared with TTTTCNQ. At a somewhat lower cast temperature, (say $\mathrm{T}_{3}$ on Figure 2) the solvent evaporation rate is still high enough but the crystallization process starts too late and only a small part of the dissolved complex crystallize. This is illustrated in Figure 1b which shows the micrograph of a sample obtained at $75^{\circ} \mathrm{C}$. Though the crystals satisfactory, from the stand point of electrical properties, the dendritic structure shows that only a small portion of the additives could crystallize. ${ }^{4}$

To overcome these difficulties, we performed the experiment shown schematically in Figure 3 (solid line). The thickening of the cast solution was at first carried out at a higher temperature (curve $T_{4}$ on Figure 2) to avoid early precipitation of the complex. The solution was then quickly cooled. The solution at a lower temperature (curve $T_{2}$ on Figure 3) became supersaturated so that low molecular weight additives rapidly crystallize in nonequilibrium dendritic forms. Since the already grown microcrystals acted as nucleation centers for further crystallite growth, a local correlation of neighbouring crystallites appeared. A relatively high viscosity of the solution at low temperatures made this possible. Micrographs of the film samples obtained in this way are shown in Figures $4 \mathrm{a}$ and $4 \mathrm{~b}$ for polycarbonate and polystyrene matrices respectively. The specific conductivity of these samples, as

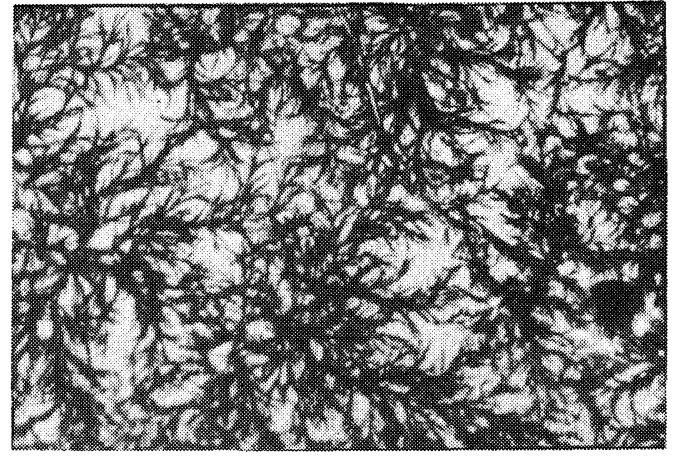

Figure 5. Micrograph of PC film containing $2 \mathrm{wt} \%$ of TTF-TCNQ prepared by rapid evaporation of the solvent under reduced pressure and crystallization at $30^{\circ} \mathrm{C}$.

determined using four-probe technique, ranged about $10^{-3}(\Omega \mathrm{cm})^{-1}$ (polycarbonate) and $10^{-2}$ $(\Omega \mathrm{cm})^{-1}$ (polystyrene).

The other possibility for creating proper crystallization conditions can be achieved by acceleration of solvent evaporation at lower temperatures through reduction of the pressure over the cast solution. These conditions are illustrated by the curve $T_{2}{ }^{\prime}$ in Figure 3. Rapid solvent evaporation at low temperatures leads to supersaturation of the solution and then to instantaneous crystallization of the CT complex. The micrograph of the PC film sample obtained in this way is shown in Figure 5.

The films prepared in this manner exhibit a specific conductivity about $10^{-2}(\Omega \mathrm{cm})^{-1}$. It should be noticed, however, that such polymer films are of rather poor quality and the results are not as well 
reproducible as before.

\section{CONCLUSIONS}

The above model concept of reticulated crystallization of highly conducting CT complexes in polymeric matrices is supported by the micrographs of films obtained under different crystallization conditions. It should be mentioned that this model concept has a qualitative meaning and is a guide line for choosing the proper conditions for crystallization processes in these systems. A quantitative theory based on our data could be formulated but it would contain many experimental parameters se- verely limiting its general application. Further research in this regard is now in progress.

\section{REFERENCES}

1. J. K. Jeszka, J. Ulański, and M. Kryszewski, Nature, 289, 390 (1981).

2. J. Ulański, J. K. Jeszka, and M. Kryszewski, Polym. Plast. Technol. Eng., 17, 139 (1981).

3. M. Kryszewski, "Semiconducting Polymers," PWNPolish Scientific Publishers, Warsaw, 1980.

4. J. K. Jeszka, J. Ulański, and M. Kryszewski, Proceedings of 27th International Symposium on Macromolecules, Abstracts, Vol. 2, Strasbourg, 1981, p 1174. 